\title{
A Statistical Sensitivity Analysis Method Using Weighted Empirical Distribution Function
}

\author{
Kiyoharu Tagawa \\ Department of Informatics, School of Science and Engineering, Kindai University \\ 3-4-1 Kowakae, Higashi-Osaka 577-8502, Japan \\ Corresponding Author: tagawa@info.kindai.ac.jp
}

\begin{abstract}
In order to estimate the q-quantile for the random variable following an unknown probability distribution, the empirical distribution function is composed from samples obtained by Monte Carlo simulation. However, the multiple sampling of Monte Carlo simulation is time-consuming in many real-world problems. Therefore, this paper proposes a new method of the computational statistics called the weighted empirical distribution function that can be used to estimate the above q-quantile from relatively few samples. The proposed method is demonstrated through a test problem and a real-world problem, namely, the sensitivity analysis of an impedance matching circuit for SAW filter.
\end{abstract}

Keywords: Computational statistics, empirical distribution, prediction interval, sensitivity analysis, SAW filter.

\section{Introduction}

In order to make an estimate, forecast or decision in real-world problems, a wide range of uncertainties have to be taken into account. Sensitivity analysis is the study of how the uncertainty in the output of a model can be apportioned to different sources of uncertainties in the model input ${ }^{(1)}$. In the engineering field, various sensitivity analysis methods have been proposed ${ }^{(2-4)}$. In the traditional sensitivity analysis methods, the sensitivity is defined based on the derivative $\partial y / \partial x$ where $y$ and $x$ denote the output (or response) and the input (or parameter) of a model. Actually, the derivative is often approximated by using the differentiation. The traditional sensitivity analysis methods have some drawbacks. For example, the model is needed to be linearized. Besides, the sensitivity is represented by the relative ratio but not measured by the absolute amount.

The statistical sensitivity analysis is a novel method that utilizes the computer algorithms of the computational statistics $^{(5)}$ for analyzing a model statistically. Besides, the Monte Carlo simulation is fundamental for the statistical sensitivity analysis. In the statistical sensitivity analysis method, the sensitivity is measured by the probability.

In this paper, the relationship between the probabilistic input and output variables of a model is considered. It is supposed that the input of the model is a set of random variables following an established probability distribution, while the model output is a random variable following an unknown probability distribution. In order to estimate the q-quantile of the latter random variable, or the output, the Empirical Cumulative Distribution Function (ECDF) ${ }^{(5)}$ is composed from a set of samples obtained by Monte Carlo simulation. The ECDF is a proper method to approximate an unknown Cumulative Distribution Function (CDF) from samples. The accuracy of ECDF depends on the number of samples. However, the multiple sampling based on Monte Carlo simulation is very time-consuming in many real-world problems. Therefore, in order to approximate an unknown CDF from relatively few samples, this paper proposes a new method named the Weighted Empirical Cumulative Distribution Function (W_ECDF).

The rest of this paper is organized as follows. In Section 2, the conventional $\mathrm{ECDF}^{(5)}$ and the proposed W_ECDF are described. In order to demonstrate the performance of the W_ECDF, it is compared with the ECDF through a test problem in Section 3. In Section 4, the proposed method is applied to a real-world problem, namely, the sensitivity analysis of an impedance matching circuit for Surface Acoustic Wave (SAW) filter. Section 5 presents conclusions and some insights to future work. 


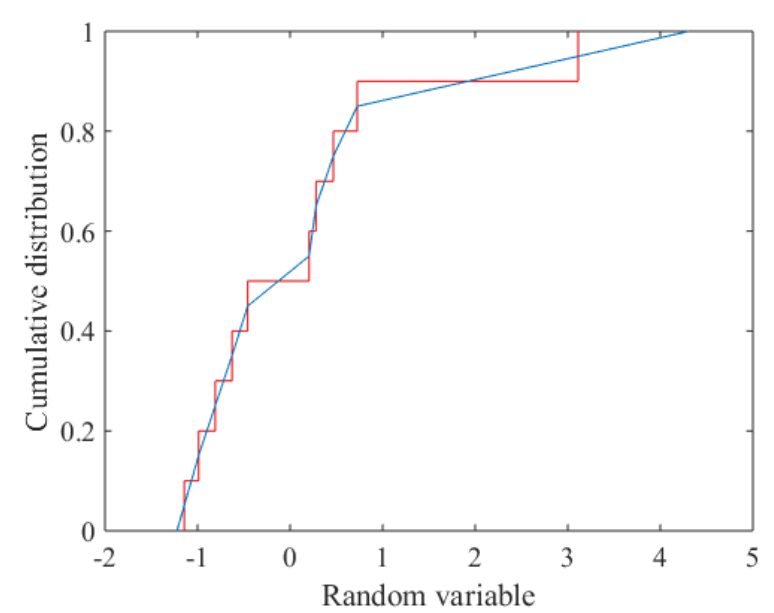

Fig. 1. Example of ECDF and smoothed ECDF

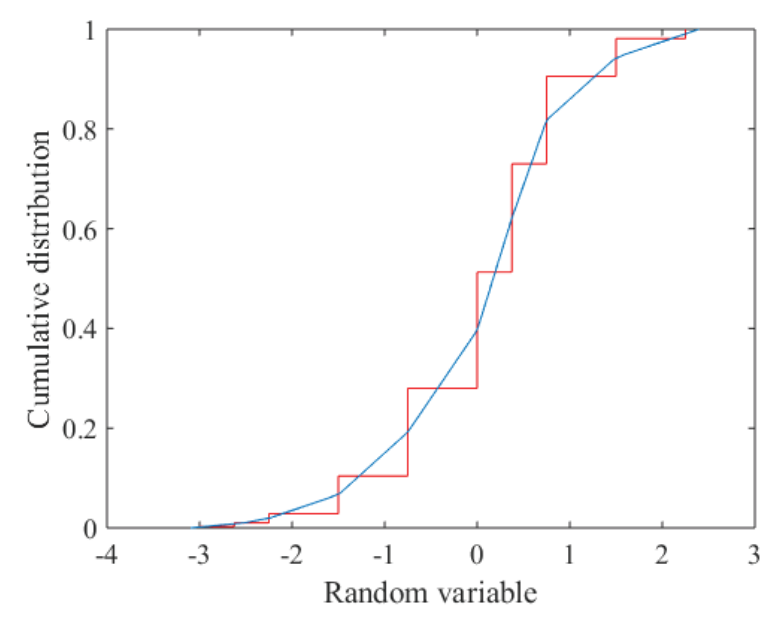

Fig. 2. Example of W_ECDF and smoothed W_ECDF

\section{Statistical Sensitivity Analysis}

\subsection{Problem Formulation}

As mentioned above, the relationship between the probabilistic input and output variables of a model is considered. Let $\vec{X} \in R^{D}$ be a vector of random variables following an established probability distribution. The vector of random variables is an input of the considering model that can be represented by a function $h: R^{D} \rightarrow R$ as

$$
Y=h(\vec{X})=h\left(X_{1}, X_{2}, \cdots, X_{D}\right) .
$$

The output of the model denoted by $Y$ becomes a random variable, too. The CDF of $Y$ is defined as

$$
F(y)=\operatorname{Pr}(Y \leq y) \text {. }
$$

From the inverse function of the CDF in (2), we can obtain the accurate q-quantile $(0 \leq q \leq 1)$ of $Y$ as

$$
y_{q}=F^{-1}(q)
$$

Besides, for a significance level $\alpha(0 \leq \alpha \leq 1)$, we can evaluate the prediction interval of the random variable $Y$ by using $\alpha / 2$ and $(1-\alpha / 2)$ quantiles as

$$
\operatorname{Pr}\left(F^{-1}(\alpha / 2) \leq Y \leq F^{-1}(1-\alpha / 2)\right)=1-\alpha .
$$

Both the quantile in (3) and the prediction interval in (4) provide useful information about the model in (1). For example, since $(1-\alpha) \%$ of the future observations of the random variable $Y$ will fall in the prediction interval, the worst case of the output can be evaluated statistically by using the upper bound of the prediction interval ${ }^{(6)}$.

Unfortunately, the strict CDF shown in (2) can't be derived mathematically for many real-world problems. That is because the function $h: R^{D} \rightarrow R$ in (1) is usually too complex. Therefore, we need to employ a method of the computational statistics for composing the CDF of $Y$ from a set of samples obtained by Monte Carlo simulation.

\subsection{Empirical Distribution Function}

The empirical distribution function, or ECDF, is the distributed function associated with the empirical measure of samples. ECDF is a step function that jumps up by $1 / N$ at each of the $N$ data points. From (1), each sample $Y^{n}$ of the output $Y$ is brought by a sample $\vec{X}^{n}$ of the input $\vec{X}$ such as $Y^{n}=h\left(\vec{X}^{n}\right) \quad(n=1, \cdots, N)$. Thereby, the ECDF of $Y$ is defined as follows:

$$
\left[\begin{array}{l}
F_{u}(y)=\frac{1}{N} \sum_{n=1}^{N} u\left(Y^{n} \leq y\right), \\
u\left(Y^{n} \leq y\right)= \begin{cases}1 ; & \text { if } Y^{n} \leq y, \\
0 ; & \text { otherwise. }\end{cases}
\end{array}\right.
$$

The step function in (5), or ECDF, is smoothed. Let $\widetilde{F}_{u}(y)$ be a smoothed ECDF in (5). Thereby, we can estimate the q-quantile $(0 \leq q \leq 1)$ of $Y$ as 


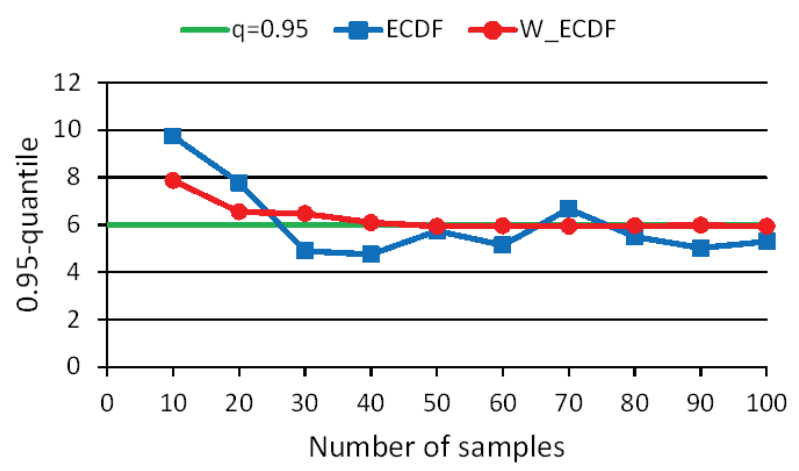

Fig. 3. Change of 0.95-quantile for sample size

$$
y_{q} \approx \widetilde{F}_{u}^{-1}(q)
$$

Figure 1 illustrates an example of the ECDF and its smoothed function composed from a set of samples of a normally distributed random variable: $Y \sim \mathbf{N}(0,1)$.

The number of samples taken from the tail part of the probability distribution of $\vec{X}$ is small. Therefore, in order to approximate $\mathrm{CDF}$ in (2) by ECDF exactly, a large number of samples $\vec{X}^{n}(n=1, \cdots, N)$ are needed.

\subsection{Weighted Empirical Distribution Function}

Let $Y^{n}=h\left(\vec{X}^{n}\right) \quad(n=1, \cdots, N)$ be a set of samples of the output $Y$ in (1). Each of the samples $Y^{n}=h\left(\vec{X}^{n}\right)$ has its own weight that is given by the

Probability Density Function (PDF) value $f\left(\vec{X}^{n}\right)$ of a corresponding sample of the input. Thereby, the weighted empirical distribution function, or W_ECDF, is defined as

$$
\left[\begin{array}{l}
F_{w}(y)=\frac{1}{W} \sum_{n=1}^{N} w\left(Y^{n} \leq y\right), \\
W=\sum_{n=1}^{N} f\left(\vec{X}^{n}\right), \\
w\left(Y^{n} \leq y\right)= \begin{cases}f\left(\vec{X}^{n}\right) ; & \text { if } Y^{n} \leq y \\
0 ; & \text { otherwise. }\end{cases}
\end{array}\right.
$$

Contrary to the ECDF defined by (5), the samples $\vec{X}^{n} \quad(n=1, \cdots, N)$ are not required to follow the probability distribution of $\vec{X}$ for the W_ECDF in (7).
Consequently, we can specify arbitrary data points for the samples $\vec{X}^{n} \quad(n=1, \cdots, N)$ of the input.

The step function in (7), or W_ECDF, is smoothed. Let $\widetilde{F}_{w}(y)$ be a smoothed W_ECDF in (7). Thereby, we can estimate the q-quantile $(0 \leq q \leq 1)$ of $Y$ as

$$
y_{q} \approx \widetilde{F}_{w}^{-1}(q)
$$

Figure 2 illustrates an example of the W_ECDF and its smoothed function that approximate the same CDF with Fig. 1. Figure 2 uses the same number of samples $N=10$ with Fig. 1. But, those samples $Y^{n}(n=1, \cdots, N)$ were given by one-dimensional Halton sequence. Besides, each sample was weighted by the PDF of $Y(Y=X)$.

\section{Test Problem}

The performance of the proposed W_ECDF in (7) is compared with the conventional ECDF in (5) through a test problem. The model of the test problem is given as

$$
Y=h\left(X_{1}, X_{2}\right)=X_{1}^{2}+X_{2}^{2} \text {. }
$$

The inputs of the model $X_{1}$ and $X_{2}$ are mutually independent random numbers following the standard normal distribution. Therefore, the output $Y$ becomes the random variable following $\chi^{2}$-distribution with two degrees of freedom. From (3), the 0.95 -quantile of $Y$ is

$$
y_{0.95}=F^{-1}(0.95)=5.991 \text {. }
$$

For composing ECDF, the input points $\left(X_{1}^{n}, X_{2}^{n}\right)$ $(n=1, \cdots, N)$ were sampled from the random numbers: $X_{1}, X_{2} \sim \mathbf{N}(0,1)$. On the other hand, for composing W_ECDF, the input points $\left(X_{1}^{n}, X_{2}^{n}\right)$ were given by two-dimensional Halton sequence and weighted by PDF.

Figure 3 shows the 0.95 -quantiles of $Y$ estimated by using the conventional ECDF and the proposed W_ECDF respectively, which depend on the sample size. From Fig. 3, we can confirm that W_ECDF estimates the 0.95-quantile in (10) accurately with fewer samples than ECDF. 


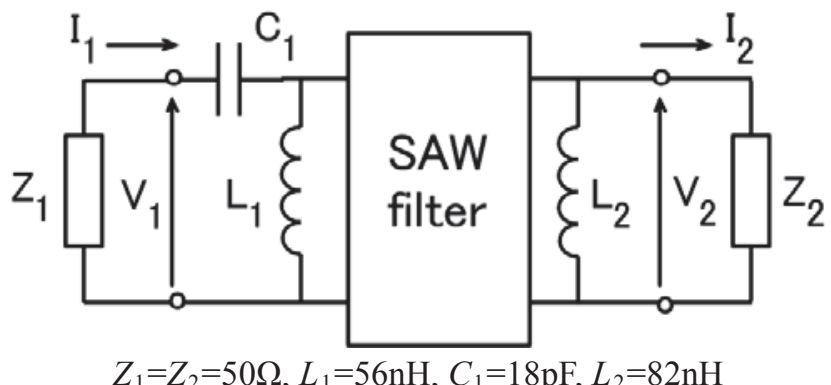

Fig. 4. Impedance matching circuit for SAW filter

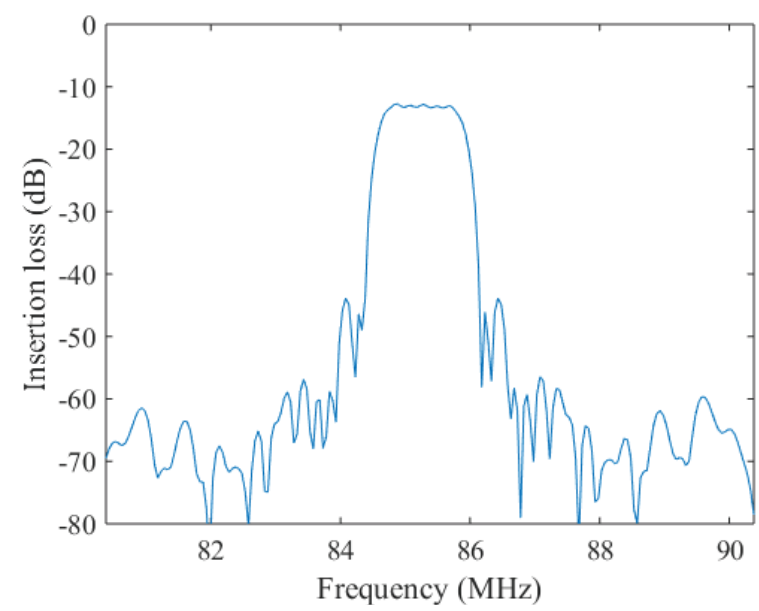

Fig. 5. Frequency response of SAW filter

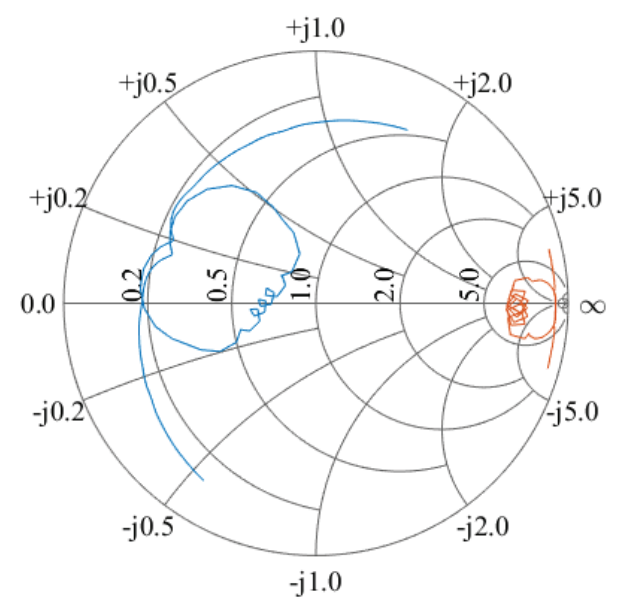

Fig. 6. Smith chart of SAW filter

\section{Sensitivity Analysis of SAW Filter}

\subsection{Impedance Matching Circuit for SAW Filter}

Surface Acoustic Wave (SAW) devices ${ }^{(7)}$ including SAW filter and SAW duplexer are widely used in the modern RF circuits of various mobile communication systems such as cellular phones. SAW devices have achieved world-wide success as mobile communication technology over the last several decades. Besides, they are studied actively to this day ${ }^{(8-10)}$. Author has been studying the computer-aided design methods of SAW filters for many years ${ }^{(11-13)}$. The proposed method is used to analyze an impedance matching circuit for SAW filter in Fig. 4.

\subsection{Network Model of SAW Filter}

The equivalent circuit model of the SAW filter shown in Fig. 4 is described by transmission line parameters: $(\hat{A}, \hat{B}, \hat{C}, \hat{D})^{(14)}$. From those parameters, the transmission line parameters $(A, B, C, D)$ of the SAW filter with the impedance matching circuit in Fig. 4 can be derived as

$$
\begin{aligned}
{\left[\begin{array}{l}
V_{1} \\
I_{1}
\end{array}\right] } & =\left[\begin{array}{cc}
1 & \frac{1}{j \omega C_{1}} \\
0 & 1
\end{array}\right]\left[\begin{array}{cc}
1 & 0 \\
\frac{1}{j \omega L_{1}} & 1
\end{array}\right]\left[\begin{array}{cc}
\hat{A} & \hat{B} \\
\hat{C} & \hat{D}
\end{array}\right]\left[\begin{array}{cc}
1 & 0 \\
\frac{1}{j \omega C_{2}} & 1
\end{array}\right]\left[\begin{array}{l}
V_{2} \\
I_{2}
\end{array}\right] \\
& =\left[\begin{array}{ll}
A & B \\
C & D
\end{array}\right]\left[\begin{array}{l}
V_{2} \\
I_{2}
\end{array}\right]
\end{aligned}
$$

where $V_{1}$ and $I_{1}$ are the voltage and current at the input port of the impedance matching circuit, while $V_{2}$ and $I_{2}$ are the voltage and current at the output port as shown in Fig. 4.

The transmission line parameters derived in (11) can be transformed into scattering parameters as

$$
\left[\begin{array}{l}
S_{11}=\frac{A Z_{2}+B-C Z_{1} Z_{2}-D Z_{1}}{A Z_{2}+B+C Z_{1} Z_{2}+D Z_{1}}, \\
S_{21}=\frac{2 \sqrt{Z_{1} Z_{2}}}{A Z_{2}+B+C Z_{1} Z_{2}+D Z_{1}}, \\
S_{12}=\frac{2(A D-B C) \sqrt{Z_{1} Z_{2}}}{A Z_{2}+B+C Z_{1} Z_{2}+D Z_{1}}, \\
S_{22}=\frac{-A Z_{2}+B-C Z_{1} Z_{2}+D Z_{1}}{A Z_{2}+B+C Z_{1} Z_{2}+D Z_{1}}
\end{array} .\right.
$$

By using the scattering parameters in (12), the network model of the SAW filter including the impedance matching circuit in Fig. 4 can be described as

$$
\left[\begin{array}{l}
b_{1} \\
b_{2}
\end{array}\right]=\left[\begin{array}{ll}
S_{11} & S_{12} \\
S_{21} & S_{22}
\end{array}\right]\left[\begin{array}{l}
a_{1} \\
a_{2}
\end{array}\right]
$$

where $a_{1}$ and $b_{1}$ denote the input and output signals at the input port of the circuit, while $a_{2}$ and $b_{2}$ denote the input and output signals at the output port of the circuit ${ }^{(7)}$.

The scattering parameters $S_{21}$ in (13) provides the transition characteristic from the input port to the output port. Then the insertion loss of SAW filter is defined as 


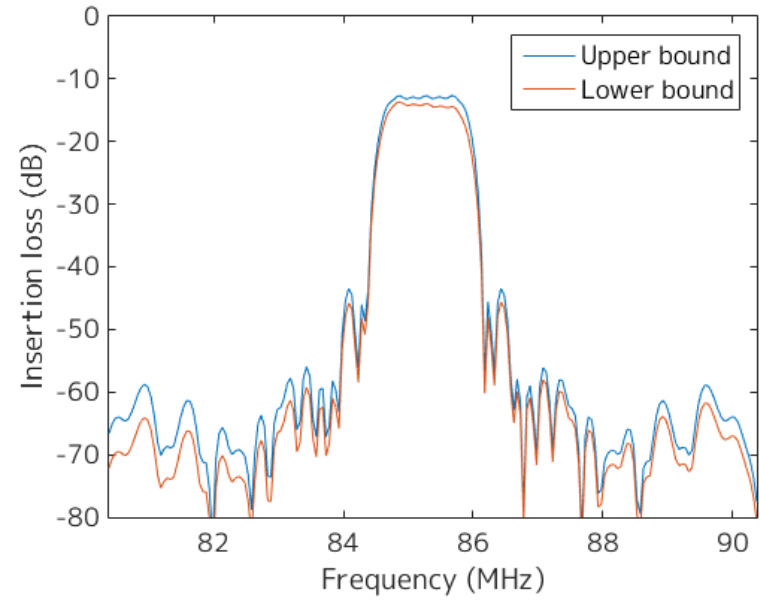

Fig. 7. Prediction interval with $\operatorname{ECDF}(k=0.05, N=100)$

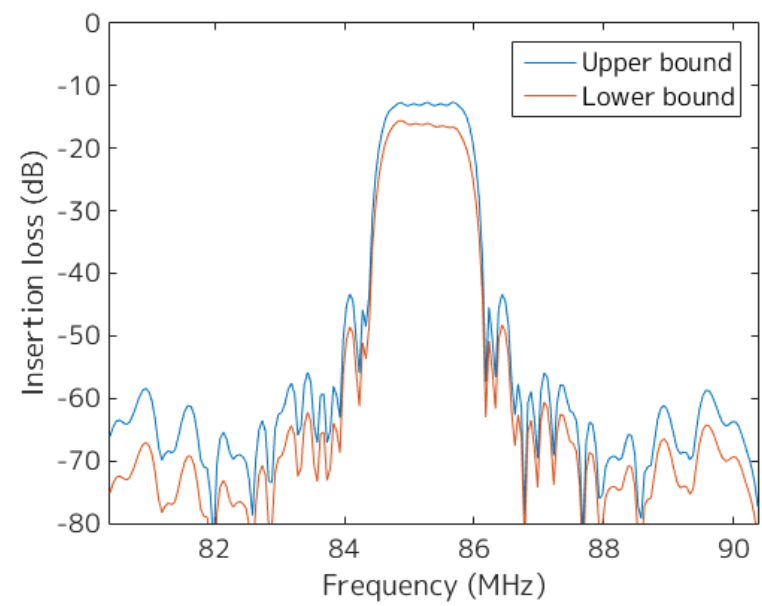

Fig. 8. Prediction interval with $\operatorname{ECDF}(k=0.1, N=100)$

$$
\text { Loss }=20 \log _{10}\left(\left|S_{21}\left(\omega, L_{1}, C_{1}, L_{2}\right)\right|\right) \text {. }
$$

The value $S_{21}$ depends on the frequency $\omega$ and the three electric elements of the impedance matching circuit: the inductances $L_{1}$ and $L_{2}$, and the capacitance $C_{1}$. Figure 5 shows the frequency response of the SAW filter, or the insertion loss in (14), with nominal values of $L_{1}, C_{1}$, and $L_{2}$ shown in Fig. 4. Incidentally, Fig. 6 shows the Smith chart of the SAW filter in which the blue line denotes the input-port and the red line denotes the output-port.

Actually, the values of the three electric elements, namely $L_{1}, C_{1}$, and $L_{2}$, fluctuate due to environmental condition and processing error. Therefore, we try to analyze the effect of the perturbations in the electric elements on the insertion loss in (14). Strictly speaking, the formula in (14) is extended into an instance of the model in (1). We suppose that the values of the three electric elements are mutually independent normal random variables as follows:

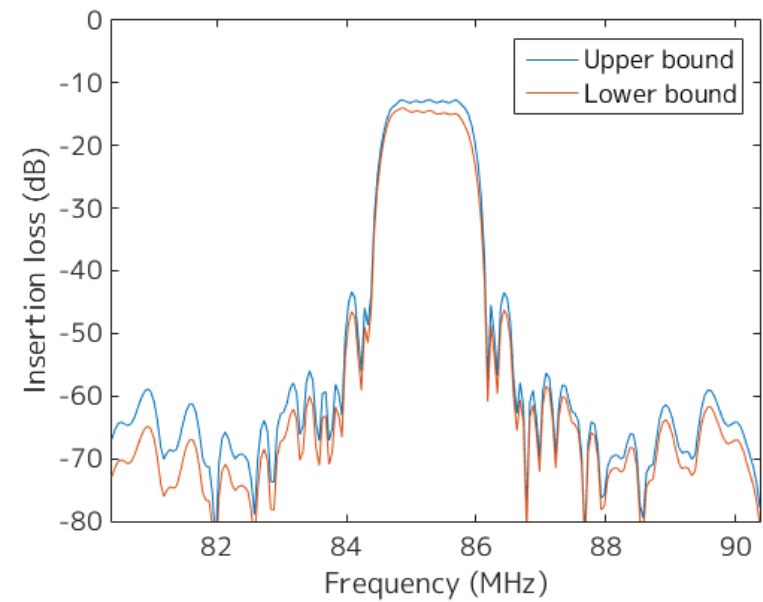

Fig. 9. Prediction interval with W_ECDF $(k=0.05, N=100)$

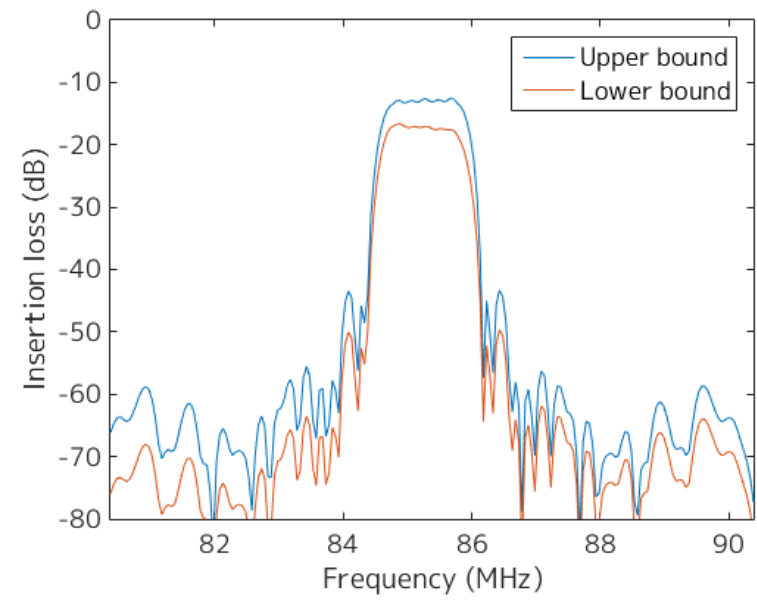

Fig. 10. Prediction interval with W_ECDF $(k=0.1, N=100)$

$$
\left[\begin{array}{l}
L_{1} \sim \mathbf{N}\left(L_{1}^{*},\left(k L_{1}^{*}\right)^{2}\right), \\
C_{1} \sim \mathbf{N}\left(C_{1}^{*},\left(k C_{1}^{*}\right)^{2}\right), \\
L_{2} \sim \mathbf{N}\left(L_{2}^{*},\left(k L_{2}^{*}\right)^{2}\right),
\end{array}\right.
$$

where $L_{1}{ }^{*}=56 \mathrm{nH}, C_{1}{ }^{*}=18 \mathrm{pF}$, and $L_{2}{ }^{*}=82 \mathrm{nH}$.

\subsection{Result of Statistical Sensitivity Analysis}

From the three random variables given by (15), the prediction interval defined in (4) was calculated with a significance level $\alpha=0.1$ for the insertion loss in (14).

Figures 7 and 8 show the prediction intervals of the insertion loss obtained by using ECDF with $N=100$ for different variance factors, namely $k=0.05$ and $k=0.1$, at each frequency point $\omega$ in (14). Similarly, Figs. 9 and 10 show the prediction intervals obtained by using W_ECDF. For W_ECDF, the input points $\left(L_{1}^{n}, C_{1}^{n}, L_{2}^{n}\right)(n=1, \cdots, N)$ were given by three-dimensional Halton sequence. 
From Figs. 7 and 9, W_ECDF provides wider prediction intervals than ECDF for the same variance factor $k=0.05$. Similarly, from Figs. 8 and 10, W_ECDF provides wider prediction intervals than ECDF for the same variance factor $k=0.1$. Especially, the lower bounds of the prediction intervals with W_ECDF are falling below the lower bounds with ECDF. That is because ECDF can't get enough number of samples $\left(L_{1}^{n}, C_{1}^{n}, L_{2}^{n}\right)$ from the tail part of the three-dimensional normal distribution shown in (15).

Comparing Fig. 9 with Fig. 10, we can confirm that the uncertainty of the insertion loss increases in proportion to the amount of the perturbations in the electric elements.

\section{Conclusions}

In order to estimate the q-quantile for the random variable following an unknown probability distribution, a new method named W_ECDF was proposed. From the results of experiments conducted on a test problem and a real-world problem, W_ECDF outperformed ECDF.

In our future work, a multi-dimensional W_ECDF will be developed. If we use a two-dimensional W_ECDF, we may draw the prediction interval even on the Smith chart in Fig. 6. Furthermore, we will use the q-quantile obtained by the proposed W_ECDF to measure the robustness of the solutions for various robust optimization problems ${ }^{(6)(15)}$.

\section{References}

(1) Andrea Saltelli : "Sensitivity analysis for importance assessment”, Risk Analysis, Vol. 22, No. 3, pp. 579-590, 2002.

(2) Jun-Fa Mao and Ernest S. Kuh : "Fast simulation and sensitivity analysis of lossy transmission lines by the method of characteristics", IEEE Trans. on Circuit and Systems - 1: Fundamental Theory and Applications, Vol. 44, No. 5, pp. 391-401, 1997.

(3) Xiaying Zhu and Natalia K. Nikolova : "Accuracy improvement of the S-parameter adjoint sensitivity analysis for shape parameters", Proceedings of IEEE International Microwave Symposium (IMS 2009), pp. 529-532, 2009.

(4) Josef Dobes, Jan Divin, Jiri Svaton, and Frantisek Vejrazka : "Using the sensitivity analysis of the noise spectral density and the sensitivity analysis of the noise figure for practical circuit design", Proceedings of IEEE International Symposium on Circuit and System
(ISCAS 2014), pp. 1676-1679, 2014.

(5) Wendy L. Martinez and Angel R. Martinez : Computational Statistics Handbook with MATLAB ${ }^{\circledR}$, Second Edition, Chapman \& Hall/CRC, 2008.

(6) Kiyoharu Tagawa : "Worst case optimization using Chebyshev inequality", Proceedings of The 7th International Conference on Bioinspired Optimization Methods and Their Applications, pp. 173-185, 2016.

http://bioma.ijs.si/conference/2016/\#schedule

(7) Ken-ya Hashimoto : Surface Acoustic Wave Devices in Telecommunications, Springer, 2000.

(8) Thomas Bauer, Christoph Eggs, Karl Wagner, and Peter Hagn : "A bright outlook for acoustic filtering", IEEE Microwave Magazine, Vol. 16, Issue 7, pp. 73-81, 2015.

(9) Roberto Gomez-Garcia, Dimitra Psychogiou, Raul Loeches-Sanchez, and Dimitrios Peroulis : "Hybrid surface acoustic wave / micro-strip signal interference bandpass filters", IET Microwaves, Antennas \& Propagation, Vol. 10, Issue 4, pp. 426-434, 2016.

(10) Shinpei Oshima, Tomonori Oshima, and Koji Wada : "A design method of matching circuit for a compact diplexer using SAW filters", Proceedings of International Conference on Electronics Packaging (ICEP 2016), pp. 23-26, 2016.

(11)Kiyoharu Tagawa and Mikiyasu Matsuoka : "Optimum design of surface acoustic wave filters based on the Taguchi's quality engineering with a memetic algorithm", Proceedings of The 9th International Conference on Parallel Problem Solving from Nature 2006, LNCS 4193, Springer, pp. 292-301, 2006.

(12) Kiyoharu Tagawa, Yukinori Sasaki, and Hiroyuki Nakamura : "Optimum design of balanced SAW filters using multi-objective differential evolution", Proceedings of The 8th International Conference on Simulated Evolution and Learning 2010, LNCS 6457, Springer, pp. 466-475, 2010.

(13)Kiyoharu Tagawa and Shoichi Harada : "Multi-noisyhard-objective robust design of balanced surface acoustic wave filters based on prediction of worst-case performance", Proceedings of EvoApplications 2015, LNCS 9028, Springer, pp. 173-185, 2015.

(14) http://www.catnet.ne.jp/triceps/cdr/cd005.

(15) Shaul Salomon, Gideon Avigad, Peter J. Fleming, and Robin C. Purshouse : "Active robust optimization: enhancing robustness to uncertain environments", IEEE Trans. on Cybernetics, Vol. 44, No. 11, pp. 2221-231, 2014. 